\title{
Mortality Rates in Users of Typical and Atypical Antipsychotics: A Database Study in Poland
}

\author{
Pawel Zagozdzon $^{1}$ (1) $\cdot$ Bartosz Goyke $^{2} \cdot$ Magdalena Wrotkowska $^{1}$
}

Published online: 15 August 2016

(c) The Author(s) 2016. This article is published with open access at Springerlink.com

\begin{abstract}
Background The role of antipsychotics in influencing mortality of patients with mental disorders is still unexplained.

Objective The aim of this study was to determine mortality rates of patients treated with atypical and typical antipsychotics and to compare these data with the mortality rates for the general population.

Methods The study was based on the 2008-2012 prescription drug reimbursement data from the Polish National Health Fund in Gdansk and mortality data from the death registry. Age-standardized death rates (SDRs) and $95 \%$ confidence intervals (CIs) were calculated for individuals prescribed solely atypical or typical antipsychotics, patients prescribed both atypical and typical antipsychotics, and patients prescribed clozapine.

Results Between 2008 and 2012, typical and/or atypical antipsychotics and clozapine were prescribed to a total of 81,313 patients. The SDR for typical antipsychotic users (69.6 per 1000, $95 \%$ CI 67.64-71.56) was higher than for those treated with both typical and atypical antipsychotics (53.25 per 1000, $95 \%$ CI 50.8-55.69) or clozapine (65.11 per $1000,95 \%$ CI 58.63-71.58). The lowest mortality was documented in the case of patients treated exclusively with atypical antipsychotics (SDR $=48.38$ per $1000,95 \% \mathrm{CI}$, 44.78-51.98). The SDRs for patients treated with antipsychotics were more than tenfold higher than the respective
\end{abstract}

Pawel Zagozdzon

pzagoz@gumed.edu.pl

1 Department of Hygiene and Epidemiology, Medical University of Gdansk, 7 Debinki Str., Gdańsk 80-211, Poland

2 National Health Fund, Gdansk, Poland
SDRs for the general population in 2008, but later in 2012, the differences dropped to threefold.

Conclusion Although the study was based on administrative record linkage and therefore could not be adjusted for potential confounders, its results suggest that mortality in atypical antipsychotic users is lower than in typical antipsychotic users.

\section{Key Points for Decision Makers}

The effect of antipsychotics on mortality in schizophrenia is highly debated. A database of refunded prescriptions created by the National Health Fund in 2008 in Poland provided an opportunity to monitor and analyze mortality rates in users of typical and atypical antipsychotics.

The lowest mortality was documented in patients treated exclusively with atypical antipsychotics compared with users of typical drugs and clozapine users.

Changes in disparities between the mortality of antipsychotic users and in the general population between 2012 and 2008 may reflect changes in physicians' prescribing behavior in Poland.

\section{Introduction}

Nearly all mental disorders are associated with increased mortality [1]. This phenomenon is observed for all of the main causes of death [2]. 
While all-cause mortality of patients with schizophrenia is two to threefold higher compared with the general population, mortality as a result of natural causes and suicide mortality are estimated to be two and ten times higher, respectively [3,4]. The growing gap in survival of patients with schizophrenia and in individuals from the general population was also documented in recent population-based cohort studies [5]. The role of antipsychotics in influencing the mortality of schizophrenia patients is still unexplained [6]. Several studies, including the landmark Clinical Antipsychotic Trials of Intervention Effectiveness (CATIE) study, showed limited differences between the various antipsychotics [7, 8]. Long-term treatment with antipsychotics is associated with lower mortality compared with no antipsychotic use and the lowest mortality was observed for clozapine [5]. Although some authors showed that the mortality risk among the second-generation antipsychotic users is lower than in persons treated with the first-generation antipsychotics, other researchers did not observe this phenomenon [9, 10]. Much of the difference is attributed to the higher prevalence of cardiovascular and metabolic disease and also other behavioral risk factors. It is not entirely clear how much of the underlying cardiovascular disease is due to the metabolic side effects of medications such as olanzapine and clozapine. The risk of QT prolongation and subsequent arrhythmia-related events is viewed as one of the most important safety aspects for atypical antipsychotics [11]. Second-generation antipsychotics, also called atypical antipsychotics, are used in many countries including Poland in the treatment of bipolar disease. Atypical antipsychotics are also prescribed to the elderly to treat behavioral and psychosocial symptoms associated with dementia and delirium, which are disorders associated with high mortality. Warning letters were issued by the US Food and Drug Administration and others about the increasing mortality associated with the use of antipsychotics in the elderly with dementia [12]. The role of antipsychotics in these diseases is still unexplained. Mentally ill patients show an intrinsic disease-related increase in medical morbidity and mortality, independent of treatment. The extent to which treatment with antipsychotics decreases or increases this intrinsic disease-related increase in medical morbidity and mortality remains unclear. To the best of our knowledge, the mortality of Polish patients with mental disorders has not been studied to date. Consequently, the aim of this study was to determine the mortality rates of patients from Northern Poland treated with atypical and typical antipsychotics and to compare these data with the mortality rates for the general population of Poland.

\section{Methods}

The study was based on the prescription drug reimbursement data from the Pomeranian Branch of the National Health Fund (NHF) for the period between January 1, 2008 and December 31, 2012. The NHF is a state institution that finances healthcare benefits from contributions paid by people insured in this organization. The NHF is responsible for the provision of healthcare benefits for all insured persons and their family members. According to the Law of 23 January, 2003 on the General Insurance in the National Health Fund (Official Journal of Law 03.45.391, with later amendments), persons covered by the general health insurance (on a compulsory or voluntary basis) are entitled to free healthcare services in the territory of Poland. More than $93 \%$ of Polish citizens are entitled to the prescription drug and health service reimbursement in Poland.

\subsection{Reimbursement Regulations}

Antipsychotics are used in the treatment of schizophrenia, psychotic disorders, and bipolar affective disorder. According to the NHF policy, atypical antipsychotics were reimbursed only for patients with schizophrenia (during the whole analyzed 2008-2012 period), bipolar affective disorder (2010-2012), and dementia (since 2011). Typical antipsychotics were reimbursed for individuals with a vast array of various psychiatric conditions including not only schizophrenia but also depression, anxiety, and psychotic organic disorders. The restricted reimbursement policy for atypical antipsychotics is associated with their higher cost and the limited resources of the Polish healthcare system. In line with the new Reimbursement Act of 2012, the spectrum of psychiatric conditions eligible for the reimbursement of antipsychotics, especially atypical antipsychotics, underwent further dramatic changes. The Reimbursement Act provisions caused increased patient charges and changed physicians' drug-prescribing behavior. Owing to a fear of being fined, physicians more often prescribe drugs fully covered by the patient.

\subsection{Study Population}

We analyzed the data for all the antipsychotic prescriptions that have been reimbursed by the NHF during a 5-year period between 2008 and 2012. Therefore, the study population included all individuals who had been prescribed at least one reimbursed antipsychotic. All the subjects were 18 years of age or older. The date of the first prescription was considered the date of the subject's entry into the study. Mortality data were obtained from the Death Registry as of December 31, 2012. Data derived from the 
Pomeranian Branch of the NHF were used, as the availability of the whole nationwide data is still limited owing to differences in informatics systems and different legal interpretations of regulations across different NHF branches.

\subsection{Statistical Analysis}

Age-standardized death rates (SDRs, with $95 \%$ confidence intervals) were calculated, cumulatively for the 2008-2012 period and separately for each year. Four groups of patients were considered: (1) individuals prescribed solely atypical antipsychotics (amisulpride, aripiprazole, quetiapine, olanzapine, risperidone, sertindole, tiapride, and ziprasidone), (2) persons prescribed solely typical antipsychotics (chlorpromazine, chlorprothixene, flupentixol, haloperidol, levomepromazine, perazine, perphenazine, prochlorperazine, promazine, sulpiride, zuclopenthixol, and clopenthixol), (3) subjects prescribed both typical and atypical antipsychotics, and (4) those prescribed clozapine. Clozapine was analyzed separately as a specific agent used in drug-resistant schizophrenia. Moreover, the respective SDRs for a geographically matched population from Pomeranian Voivodeship were calculated.

\section{Results}

A total of 1,095,518 prescriptions were issued for 84,881 patients during the analyzed period. The prevalence of getting a prescription for an antipsychotic was $0.9 \%$. Characteristics of the 81,313 typical and/or atypical antipsychotic and clozapine users are presented in Table 1. The groups of patients prescribed atypical antipsychotics, alone $(n=9767)$ or in combination with the typical antipsychotics $(n=11,114)$, were younger and included a higher proportion of women than the group treated solely with typical antipsychotics $(n=56,977)$. Moreover, clozapine users $(n=3455)$ were younger than the remaining patients. The number of refunded prescriptions dramatically dropped after 2008. There was only $40 \%$ of baseline prescriptions observed in 2009 and $25 \%$ in 2012 .

The proportion of patients who died during the analyzed period was $21 \%$ for typical antipsychotic users, $17.1 \%$ for atypical antipsychotic users, $16.1 \%$ for atypical and typical neuroleptic users, and $16.1 \%$ for clozapine users. The SDRs for all the analyzed groups are shown in Table 2 . The SDR for typical antipsychotic users was higher than for those treated with typical and atypical antipsychotics or clozapine. Irrespective of sex, the lowest mortality was documented in patients treated exclusively with atypical antipsychotics.

The most evident differences in the SDRs for the antipsychotic users and the general population was observed in 2008 and 2009. Overall, 2008-2012 SDRs for female and male patients from the analyzed groups are presented in Figs. 1 and 2, and the SDRs for year 2012 separately in Figs. 3 and 4.

The difference in the SDRs for the typical and atypical antipsychotic users was more pronounced in the case of men than in women. During the whole 2008-2012 period, the SDRs for the clozapine users were higher than for the atypical antipsychotic users (Figs. 1,2). The SDR for male clozapine users was lower than for men treated with typical antipsychotics (Fig. 2). Women prescribed clozapine and those treated with conventional antipsychotics did not differ significantly in terms of their SDRs (Fig. 1). In 2012, the absolute differences in the SDRs for patients from various groups were less pronounced than in 2008-2011 (Figs. 3, 4). Treatment with both atypical and typical antipsychotics was associated with a slightly higher mortality than with the atypical antipsychotics alone.

Table 1 Characteristics of typical and/or atypical antipsychotic and clozapine users

\begin{tabular}{|c|c|c|c|c|c|}
\hline Parameter & $\begin{array}{l}\text { Only typical } \\
\text { antipsychotics }\end{array}$ & $\begin{array}{l}\text { Only atypical } \\
\text { antipsychotics }\end{array}$ & $\begin{array}{l}\text { Typical and atypical } \\
\text { antipsychotics }\end{array}$ & Clozapine & $p$ value \\
\hline 2008-2012 (n) & 56,977 & 9767 & 11,114 & 3455 & \\
\hline $2008(n)$ & 24,626 & 3897 & 7659 & 2658 & \\
\hline $2009(n)$ & 10,279 & 1306 & 1368 & 310 & \\
\hline $2010(n)$ & 8507 & 1289 & 943 & 221 & \\
\hline $2011(n)$ & 7510 & 1480 & 743 & 171 & \\
\hline $2012(n)$ & 6055 & 1795 & 410 & 95 & \\
\hline Age, years $($ mean $\pm S D)$ & $57.68 \pm 18.47$ & $55.98 \pm 21.74$ & $54.04 \pm 19.02$ & $50.95 \pm 18.78$ & $<0.001$ \\
\hline Male patients, $\%(n)$ & $43.96(25,043)$ & 39.80 (3887) & $42.32(6410)$ & $50.22(1720)$ & $<0.001$ \\
\hline Deaths, \% (n) & $21.02(11,976)$ & $17.12(1672)$ & $16.13(1793)$ & $16.15(558)$ & $<0.001$ \\
\hline
\end{tabular}

SD standard deviation 
Table 2 Age-standardized death rates per 1000 (with $95 \%$ confidence intervals) for patients treated with typical and/ or atypical antipsychotics or clozapine and individuals from the general population

\begin{tabular}{|c|c|c|c|}
\hline Group & Women & Men & Total \\
\hline \multicolumn{4}{|l|}{ 2008-2012 } \\
\hline Only atypical agents & $39.06(34.79-43.33)$ & $62.92(56.6-69.17)$ & $48.38(44.78-51.98)$ \\
\hline Atypical + typical agents & $42.82(39.83-45.8)$ & $68.4(64.34-72.45)$ & $53.25(50.8-55.69)$ \\
\hline Clozapine & $53.5(45.69-61.32)$ & $79.2(69.29-89.11)$ & $65.11(58.63-71.58)$ \\
\hline Only typical agents & $49.51(46.0-52.03)$ & 94.68 (91.65-97.71) & $69.6(67.64-71.56)$ \\
\hline General population & $5.42(5.37-5.47)$ & $10.12(10.04-10.21)$ & $7.48(7.43-7.53)$ \\
\hline \multicolumn{4}{|l|}{2008} \\
\hline Only atypical agents & $68.17(61.25-75.1)$ & $98.78(88.22-109.34)$ & $79.21(73.31-85.11)$ \\
\hline Atypical + typical agents & $72.45(66.82-78.1)$ & $102.98(95.32-110.65)$ & $84.33(79.78-88.88)$ \\
\hline Clozapine & 85.01 (70.83-99.18) & $113.99(98.17-129.82)$ & $95.57(85.28-105.87)$ \\
\hline Only typical agents & $71.56(67.2-75.91)$ & $126.31(121.21-131.4)$ & 94.44 (91.16-97.73) \\
\hline General population & $5.79(5.66-5.91)$ & 10.77 (10.56-10.97) & $7.95(7.84-8.06)$ \\
\hline \multicolumn{4}{|l|}{2009} \\
\hline Only atypical agents & $55.04(49.77-60.31)$ & $83.5(74.77-92.23)$ & $65.32(60.57-70.08)$ \\
\hline Atypical + typical agents & $58.66(54.14-63.18)$ & 92.17 (85.6-98.73) & $71.45(67.65-75.24)$ \\
\hline Clozapine & $62.99(51.68-74.3)$ & $97.65(82.37-112.92)$ & $78.07(68.25-87.88)$ \\
\hline Only typical agents & $59.91(55.56-64.27)$ & $103.56(98.77-108.35)$ & $77.45(74.29-80.61)$ \\
\hline General population & $5.62(55.01-57.4)$ & $10.56(10.36-10.76)$ & $7.76(7.65-7.87)$ \\
\hline \multicolumn{4}{|l|}{2010} \\
\hline Only atypical agents & $45.25(40.66-49.85)$ & $70.61(63.07-78.15)$ & $54.99(50.81-59.16)$ \\
\hline Atypical + typical agents & $45.73(41.98-49.48)$ & $73.8(67.92-79.68)$ & $57.18(53.78-60.59)$ \\
\hline Clozapine & $48.17(39.19-57.15)$ & 70.97 (58.03-83.92) & $58.57(50.56-66.57)$ \\
\hline Only typical agents & $45.55(42.6-48.85)$ & 83.68 (79.33-88.04) & $61.48(58.74-64.22)$ \\
\hline General population & $5.26(5.15-5.37)$ & $9.96(9.77-10.15)$ & $7.32(7.22-7.43)$ \\
\hline \multicolumn{4}{|l|}{2011} \\
\hline Only atypical agents & $22.22(19.14-25.3)$ & $32.49(27.41-37.56)$ & $26.13(23.43-28.83)$ \\
\hline Atypical + typical agents & $24.42(21.57-27.27)$ & $37.31(33.06-41.57)$ & $29.51(27.12-31.91)$ \\
\hline Clozapine & $27.67(19.5-35.85)$ & $36.19(27.1-46.48)$ & $31.08(24.99-37.17)$ \\
\hline Only typical agents & $20.6(18.4-22.8)$ & $34.52(31.36-37.69)$ & $26.95(24.95-28.95)$ \\
\hline General population & $5.15(5.04-5.27)$ & 9.57 (9.39-9.76) & $7.1(6.99-7.2)$ \\
\hline \multicolumn{4}{|l|}{2012} \\
\hline Only atypical agents & $16.2(13.77-18.62)$ & $29.61(24.71-34.51)$ & $25.36(22.46-28.27)$ \\
\hline Atypical + typical agents & $17.41(15.13-19.7)$ & $30.46(26.47-34.44)$ & $26.89(24.43-29.35)$ \\
\hline Clozapine & $22.29(15.63-29)$ & $24.81(16.39-33.23)$ & $25.98(20.35-31.61)$ \\
\hline Only typical agents & $23.57(20.15-27)$ & $40.49(37.17-43.82)$ & $27.85(25.91-29.79)$ \\
\hline General population & $5.32(5.21-5.43)$ & $9.86(9.68-10.05)$ & $7.32(7.22-7.42)$ \\
\hline
\end{tabular}

\section{Discussion}

We showed that depending on the group of antipsychotics and the analyzed year, the SDRs for Polish patients were three to more than tenfold higher than the respective SDRs for the general population. However, the difference in the mortality rates apparently decreased with time. Previous studies dealing with the problem in question showed that the mortality risk of schizophrenia patients is two to threefold higher compared with individuals from the general population [9]. We observed such a small difference solely for 2012, when the SDR for atypical antipsychotic users was threefold higher than the SDR for the general population. However, up to tenfold differences in the SDRs of antipsychotic users and the general population were observed in 2008-2009. It is very likely that not all individuals who have been prescribed antipsychotics experienced schizophrenia. Some patients eligible for treatment with typical antipsychotics may present with somatic disorders affecting mental health, or mental retardation, both associated with an increased mortality risk. Therefore, the relative excess in the mortality of patients who have been prescribed antipsychotics in 2008-2009 might result from a higher prevalence of somatic disorders in this group. This 


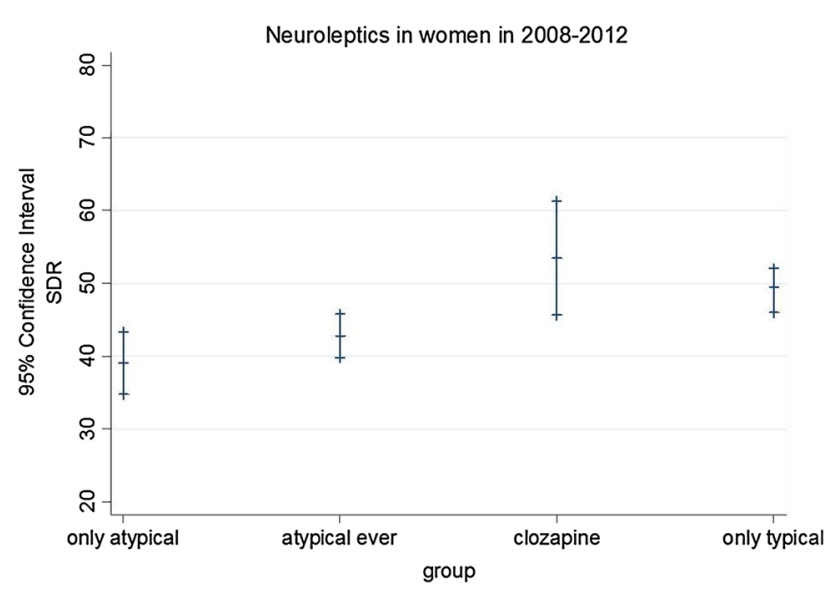

Fig. 1 Age-standardized death rates (SDRs) per 1000 with $95 \%$ confidence intervals for female patients from Northern Poland prescribed typical and/or atypical antipsychotics and clozapine; cumulated data for 2008-2012

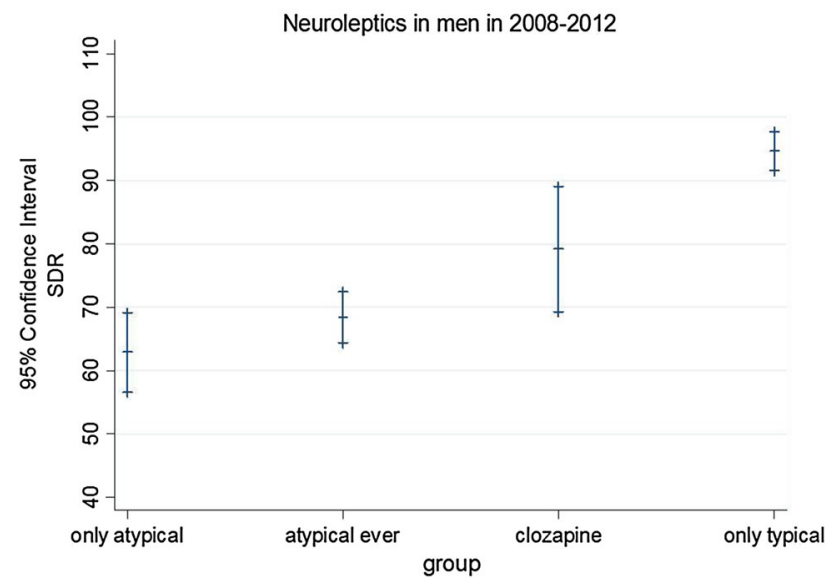

Fig. 2 Age-standardized death rates (SDRs) per 1000 with $95 \%$ confidence intervals for male patients from Northern Poland prescribed typical and/or atypical antipsychotics and clozapine; cumulated data for 2008-2012

observation is consistent with the evidence from previous studies analyzing the effect of physical illness on the mortality of patients with mental disorders [13].

In turn, the relative decrease in the mortality of antipsychotic users observed in 2012 might reflect changes in the drug reimbursement criteria and prescription practices of Polish physicians, for example, the inclusion of less severe cases as eligible for treatment or the reimbursement of atypical antipsychotics solely for patients with specific conditions. A number of physicians were sued by the NHF in 2006-2009, and eventually were sentenced to refund the costs of second-generation antipsychotic reimbursement because of inconsistencies in medical documentation. Furthermore, the Reimbursement Act of 2012 influenced the antipsychotic prescription patterns in Poland. Owing to the introduction of tight control mechanisms, the drugs are

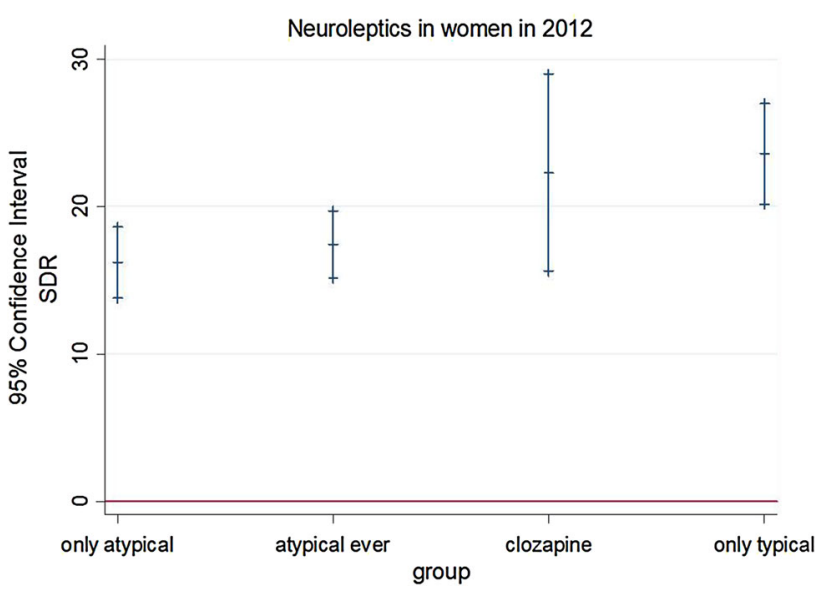

Fig. 3 Age-standardized death rates (SDRs) per 1000 with $95 \%$ confidence intervals for female patients from Northern Poland prescribed typical and/or atypical antipsychotics and clozapine; separate data for year 2012

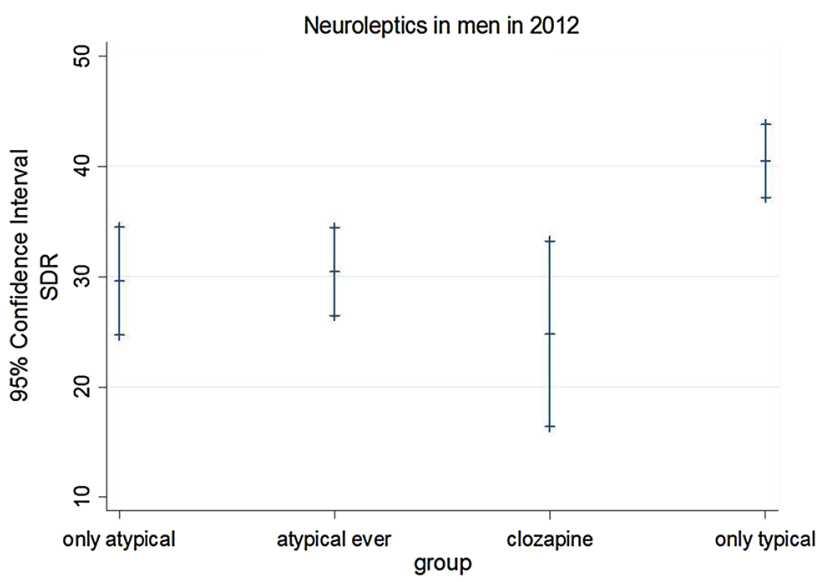

Fig. 4 Age-standardized death rates (SDRs) per 1000 with $95 \%$ confidence intervals for male patients from Northern Poland prescribed typical and/or atypical antipsychotics and clozapine; separate data for year 2012

reimbursed only in specified cases. Consequently, it is likely that in 2012, atypical antipsychotics were prescribed on-label in most cases, i.e., solely for patients with established schizophrenia or bipolar affective disorder. Therefore, only the data for 2012, when the SDRs for atypical antipsychotic users were approximately threefold higher than the SDRs for the general population, seem to be free from a potential bias, especially taking into account that the threefold excess in the mortality of patients with mental disorders was also reported previously from other countries [14]. The drop in the number of refunded prescriptions probably reflects the shift from refunded drugs to drugs fully covered by the patients.

The discrepancy in the risk difference between typical and atypical neuroleptics users can be explained by channeling bias. Channeling is a form of allocation bias, where 
drugs with similar therapeutic indications are prescribed to groups of patients with prognostic differences [15]. Claimed advantages of a newer atypical drugs may channel to patients with a special pre-existing morbidity, with the consequence that disease states can be incorrectly attributed to the use of the drug. Generally, in Europe and USA, payers do not typically dictate which antipsychotic to use, although they may give guidance that influences the changing pattern of prescriptions through time.

Potential causes for the excess in the mortality of Polish patients with mental disorders include cigarette smoking, alcohol and medication abuse, inappropriate diet, and economic deprivation. Other postulated reasons behind the higher mortality of subjects from this group include a higher prevalence of concomitant cardiovascular disorders and increased suicide risk [16].

We showed that the subjects treated with typical and atypical antipsychotics differed significantly in terms of their mortality rates. The results of the largest meta-analysis published to date suggest that some second-generation antipsychotics may be more efficient than the first-generation agents, but this finding should be interpreted cautiously, taking into account all potential limitations inherent to meta-analyses [17]. A large retrospective cohort study of elderly patients showed that the use of typical antipsychotics is associated with a significantly higher adjusted mortality risk [18]. The fact that the SDRs for individuals prescribed both typical and atypical antipsychotics were higher than the SDRs for patients treated exclusively with the atypical agents can be interpreted in terms of the greater severity of the disease in the former group, which enforced the use of polytherapy [19].

\subsection{Study Limitations}

The principal limitation of this study stems from the fact that it was based on administrative record linkage. As a result, our analysis was not adjusted for such important modifiers of mortality risk such as concomitant diseases, and psychosocial, demographic, and lifestyle-related factors, and was controlled only for the age and sex of the participants. The difficulties related to the analysis of administrative records were discussed previously [20]. The differences in the proportion of atypical antipsychotics prescribed during consecutive years might reflect a prescription and selection bias, namely selection by indication, owing to a reimbursement policy or as a result of marketing activities undertaken by a manufacturer. Prescription bias occurs when patients with systemic diseases affecting the brain secondarily are not prescribed atypical drugs because patients with organic disease and exogenous psychosis are not eligible. It is noteworthy that new indications for treatment with atypical antipsychotics (i.e., bipolar affective disorder and dementia) emerged during the study period. Finally, we do not know the proportion of Polish physicians who refrained from prescribing an atypical antipsychotic when the diagnosis was ambiguous and/or inappropriately documented.

\section{Conclusion}

Polish patients eligible for treatment with antipsychotics differed from the general population of Poland in terms of their mortality rates. The risk of death was more than tenfold higher than the respective risk for the general population in 2008 , but later in 2012 , the differences dropped to threefold. The lowest number of refunded prescriptions was observed in 2012, possibly owing to a change in the drug refund scheme. In individuals who were prescribed typical antipsychotics, the mortality rate was significantly higher than in ever users of atypical medications and people using only atypical drugs. Mortality risk in clozapine users was also higher than in users of atypical medications. The beneficial effect of atypical antipsychotic use on mortality is more pronounced in men than in women. Antipsychotics are prescribed not only to individuals with purely mental illnesses but also to persons whose mental health is affected owing to the presence of a somatic condition or psychosocial problem. Antipsychotic use may be also affected by reimbursement regulations. Consequently, the antipsychotic prescription rate should not be considered a surrogate marker for the prevalence of psychiatric disorders.

\section{Compliance with Ethical Standards}

Funding This work was supported by the Medical University of Gdansk (Grant No. ST-20).

Conflict of interest Pawel Zagozdzon, Bartosz Goyke, and Magdalena Wrotkowska declare that there were no conflicts of interest in relation to the subject of this study.

Open Access This article is distributed under the terms of the Creative Commons Attribution-NonCommercial 4.0 International License (http://creativecommons.org/licenses/by-nc/4.0/), which permits any noncommercial use, distribution, and reproduction in any medium, provided you give appropriate credit to the original author(s) and the source, provide a link to the Creative Commons license, and indicate if changes were made.

\section{References}

1. Chesney E, Goodwin GM, Fazel S. Risks of all-cause and suicide mortality in mental disorders: a meta-review. World Psychiatry. 2014;13(2):153-60. 
2. Lawrence D, Kisely S, Pais J. The epidemiology of excess mortality in people with mental illness. Can J Psychiatry. 2010;55(12):752-60.

3. McGrath J, Saha S, Chant D, Welham J. Schizophrenia: a concise overview of incidence, prevalence, and mortality. Epidemiol Rev. 2008;30(1):67-76.

4. Saha S, Chant D, McGrath J. A systematic review of mortality in schizophrenia: is the differential mortality gap worsening over time? Arch Gen Psychiatry. 2007;64(10):1123-31.

5. Tiihonen J, Lonnqvist J, Wahlbeck K, et al. 11-year follow-up of mortality in patients with schizophrenia: a population-based cohort study (FIN11 study). Lancet. 2009;374(9690):620-7.

6. Weinmann S, Aderhold V, Mueller-Oerlinghausen B. Influence of antipsychotics on mortality in schizophrenia: evidence from observational studies. Pharmacopsychiatry. 2009;42(5):246.

7. Lieberman JA, Stroup TS. The NIMH-CATIE Schizophrenia Study: what did we learn? Am J Psychiatry. 2011;168(8):770-5.

8. Leucht S, Cipriani A, Spineli L, et al. Comparative efficacy and tolerability of 15 antipsychotic drugs in schizophrenia: a multiple-treatments meta-analysis. Lancet. 2013;382(9896):951-62.

9. Auquier $\mathrm{P}$, Lancon C, Rouillon F, et al. Mortality in schizophrenia. Pharmacoepidemiol Drug Saf. 2006;15(12):873-9.

10. Weinmann S, Read J, Aderhold V. Influence of antipsychotics on mortality in schizophrenia: systematic review. Schizophr Res. 2009;113(1):1-11.

11. Raschi E, Poluzzi E, Godman B, et al. Torsadogenic risk of antipsychotics: combining adverse event reports with drug utilization data across Europe. PLos One. 2013;8(11):e81028.

12. Steinberg M, Lyketsos CG. Atypical antipsychotic use in patients with dementia: managing safety concerns. Am J Psychiatry. 2012;169(9):900-6.

13. Batty GD, Hamer M, Der G. Does somatic illness explain the association between common mental disorder and elevated mortality? Findings from extended follow-up of study members in the UK Health and Lifestyle Survey. J Epidemiol Commun Health. 2012;66(7):647-9.

14. Bushe CJ, Taylor M, Haukka J. Mortality in schizophrenia: a measurable clinical endpoint. J Psychopharmacol. 2010;24(11): $17-25$.

15. Petri H, Urquhart J. Channeling bias in the interpretation of drug effects. Stat Med. 1991;10(4):577-81.

16. De Hert M, Dekker JM, Wood D, et al. Cardiovascular disease and diabetes in people with severe mental illness position statement from the European Psychiatric Association (EPA), supported by the European Association for the Study of Diabetes (EASD) and the European Society of Cardiology (ESC). Eur Psychiatry. 2009;24(6):412-24.

17. Hartling L, Abou-Setta AM, Dursun S, et al. Antipsychotics in adults with schizophrenia: comparative effectiveness of firstgeneration versus second-generation medications: a systematic review and meta-analysis. Ann Intern Med. 2012;157(7):498511.

18. Wang P-S, Wu S-L, Ching H-Y. The use of psychiatric drugs and worsening body mass index among inpatients with schizophrenia. Int Clin Psychopharmacol. 2014;29(4):235-8.

19. Correll CU, Rummel-Kluge C, Corves C, et al. Antipsychotic combinations vs monotherapy in schizophrenia: a meta-analysis of randomized controlled trials. Schizophr Bull. 2009;35(2): 443-57.

20. Sarlon E, Millier A, Aballea S, Toumi M. Evaluation of different approaches for confounding in nonrandomised observational data: a case-study of antipsychotics treatment. Commun Ment Health J. 2014;50(6):711-20. 